A\&A 381, 761-770 (2002)

DOI: $10.1051 / 0004-6361: 20011568$

(c) ESO 2002

\title{
Environmental effects in galaxies
}

\author{
The data ${ }^{\star, \star \star}$ \\ D. F. de Mello ${ }^{1}$, M. A. G. Maia ${ }^{2}$, and T. Wiklind ${ }^{1}$ \\ 1 Onsala Space Observatory, 43992 Onsala, Sweden \\ e-mail: duilia@oso.chalmers.se, tommy@oso.chalmers.se \\ 2 Observatório Nacional, Rua Gal. José Cristino 77, RJ 20921, Brazil \\ e-mail: maia@on.br
}

Received 5 January 2001 / Accepted 15 October 2001

\begin{abstract}
We present optical and millimetric data for 47 intermediate Hubble type spiral galaxies located either in dense environments or in the field. We compare correlations between global parameters, such as far-infrared luminosity, blue luminosity, and total molecular gas content, with other samples of galaxies, including normal galaxies, clusters and ultraluminous infrared galaxies. We find that overall our sample is a well-defined subset of these other samples of galaxies.
\end{abstract}

Key words. galaxies: interactions - general - stellar content

\section{Introduction}

A longstanding issue in galaxy evolution is whether galaxies evolve according to a given set of initial conditions or whether the environment in which they reside is decisive for their evolution; i.e. whether galaxy evolution depends on nature or nurture. In order to search for environmental effects in galaxy properties, we have obtained optical and millimetric data for galaxies in dense regions of the Southern sky and in the field. In de Mello et al. (2002, hereafter Paper II) we present an extensive analysis of the data. The main results we found are: intermediate type spirals in dense environments have on average less molecular gas per blue luminosity, lower current SFR, the same SFE and higher atomic gas fraction when compared with field galaxies. Although none of the above results stand out as a single strong diagnostic, given their statistical significance (see Table 3 of Paper II), taken together they suggest a trend for diminished gas content and star formation activity in galaxies in high density environments. We also found that SFR per blue luminosity increases linearly as the total amount of gas increases in LINERs. This result, based on a small sample, suggests that LINERs are

Send offprint requests to: D. de Mello,

e-mail: duilia@oso.chalmers.se

* Based on observations at the European Southern Observatory at the $15 \mathrm{~m}$ Swedish ESO Submillimetre telescope, SEST, and at the the $1.52 \mathrm{~m}$ telescope which is operated under the ESO-ON agreement.

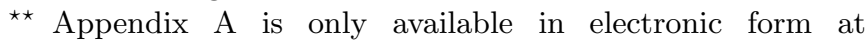
http://www.edpsciences.org powered by star formation rather than an AGN. We refer the reader to Paper II for a more detailed analysis of these results.

In this paper we present the optical and millimetric data; it is organized as follows. Section 2 describes the sample, Sect. 3 describes the optical data, Sect. 4 describes the millimetric data, Sect. 5 describes general properties, and a comparison with other samples, Sect. 6 presents a summary and conclusions. A database of optical and millimetric spectra together with digitized images are shown in Appendix A (only available in electronic form).

\section{Sample selection}

\subsection{Previous samples}

Surveys of the molecular gas content in galaxies have in general been done on samples which are far-infrared selected, or galaxies selected exclusively for belonging to clusters or groups (often with a far-infrared selection criteria on top; e.g. Casoli et al. 1991; Combes et al. 1994; Leon et al. 1998). A few exceptions exist in the literature. For example, Sage (1993) presents the CO content of a distance limited sample of 65 non-strongly interacting spiral galaxies, and Horellou et al. (1995) present a CO and HI survey of spiral and lenticular galaxies in the Fornax cluster, both based on samples selected without a far-infrared criterion.

However, until now no survey of galaxies in different environments has included a rigorously selected control sample. For instance, the sample by Casoli et al. (1998) 
which contains a large sample of 582 objects is an important source of information concerning molecular gas in spiral galaxies. However, it was built by gathering data from various surveys and is very heterogeneous in terms of morphology and environment. It contains galaxies from several clusters as well as galaxies in the field.

\subsection{Dense environment and Control Sample (HDS and CS)}

In view of these biases plagueing existing samples we have selected our sample from the catalog by Maia et al. (1994) which contains objects in low and high density areas of the Southern sky. The selection of groups adopted by Maia et al. is similar to the methodology developed by Huchra \& Geller (1982) with the adaptations described by Maia et al. (1989). The catalog was drawn from the ESO/Uppsala Survey of the ESO(B) Atlas (Lauberts 1982) and used velocity information from the Southern Sky Redshift Survey (e.g., da Costa et al. 1989). The groups are defined to be formed by the accumulation of galaxy pairs with a member in common.

- The high density sample (HDS, hereafter) is formed by galaxies that are in groups of three or more members. They have a density contrast $\delta \rho / \rho \geq 500$. This is equivalent to densities larger than 18 galaxies $/ \mathrm{Mpc}^{3}$. All the selected objects have radial velocities (after correction for Virgo infall) smaller than $8000 \mathrm{~km} \mathrm{~s}^{-1}$.

- The control sample (CS, hereafter) is made up of galaxies which are not members of any group and which are situated in regions with density contrast $\delta \rho / \rho \leq 0.01$, i.e. less than 0.0004 galaxies $/ \mathrm{Mpc}^{3}$.

\subsection{HDS versus compact groups and poor groups}

Although a group-finding algorithm was used to generate the samples, the idea is not to identify groups (either loose or compact), but galaxies in high and low local density environments. The main difference between the HDS and compact groups of galaxies is the isolation criterion which is imposed by the groups selection (Hickson 1982; Coziol et al. 2000). The only 2 compact groups (HCG 21 and HCG 90) in the region searched by Maia et al. (1994) $\left(b^{\mathrm{II}} \leq-30^{\circ}, \delta<-17.5\right)$ have 3 galaxies of each group taking part of the HDS, but none of them take part in the present subsample analysis.

The HDS should also not be confused with poor groups which are defined as systems with less than five bright galaxies but which can have 20-50 faint members (e.g., Zabludoff \& Mulchaey 1998; Willmer et al. 1999). Some galaxies in these poor groups are certainly part of the HDS, but since our selection includes only members with known redshift, the HDS will have only the brighter members which have measured redshift. The HDS and CS contain in total 151 and 179 galaxies, respectively.

\subsection{Our subsample: Morphology selection}

Maia et al. (1994) have analysed the morphology distribution of the HDS and CS and concluded that the HDS has an excess of early-type galaxies compared to the CS. This is interpreted as an effect of the morphology-density relationship (Dressler 1980); i.e. a correlation between morphological types and local density showing that the fraction of early-type galaxies increases as a function of local galaxy density while the fraction of later types decreases (see also Sanroma \& Salvador-Solé 1990; Whitmore \& Gilmore 1991). Since there are galaxies of all morphologies in the HDS and in the CS, the main goal of our work is to evaluate the effects of the environment in galaxies of the same morphological type when compared with isolated galaxies. The ideal survey would include all galaxies in the HDS and CS, however, due to large size of the samples we have imposed such a selection which is fundamental in order to avoid any bias due to the well-known correlation between morphology and physical properties of galaxies. Figures 2-4 of Roberts \& Haynes (1994) summarize clearly how morphology is correlated with fundamental properties of galaxies such as, blue luminosity, far infrared lumninosity, total mass, and neutral hydrogen mass. One of their conclusions is that, although the scatter is large, Sa-Sc have near constant molecular gas normalized either by the blue luminosity or by the total mass. They also pointed out that later-type spirals have less molecular gas and suggest that this could also be due to the $\mathrm{CO}$ to $\mathrm{H}_{2}$ conversion factor which would depend on morphology. Therefore, in order to have an homogeneous sample, we selected mostly intermediate spiral galaxies; i.e. Sb, Sbc, and Sc, avoiding $\mathrm{Sa}$ and Sd galaxies. In this work we present the analysis of the optical and millimetric data of a subsample of 47 spiral galaxies, 22 in the HDS and 25 in the CS, with velocities less than $5500 \mathrm{~km} \mathrm{~s}^{-1}$.

Table 1 lists information taken from the NASA/IPAC Extragalactic Database (NED) on each galaxy as follows. Column 1: designation in the ESO-Uppsala cata$\log$ (LV89); Col. 2: designation in other catalogs; Col. 3: right ascension $(\mathrm{h} \mathrm{m} \mathrm{s})$ and declination $\left({ }^{\circ} /\right.$ ' $)$ for J2000; Col. 4: type of sample (control sample $=$ CS and high density sample $=$ HDS) and morphological type (Lauberts \& Valentijn 1989, hereafter LV89) $1=\mathrm{Sa}, 2=\mathrm{Sa}-\mathrm{b}, 3=\mathrm{Sb}$, $4=$ Sb-c, $5=$ S..., $6=$ Sc, Sc-d, $7=$ S.. /Irr, $8=$ Sd; Col. 5 : morphological type from The Third Reference Catalogue of Bright Galaxies (RC3; de Vaucouleurs et al. 1991); Col. 6: number of galaxies in the same group (Maia et al. 1989); Col. 7: mean pairwise separation in Mpc (Maia et al. 1989); Col. 8: $B_{\mathrm{T}}$ magnitude from RC3; Col. 9: IRAS $60 \mu \mathrm{m}$ flux in Jy (Moshir et al. 1990), and Col. 10: IRAS $100 \mu \mathrm{m}$ flux in Jy (Moshir et al. 1990).

\section{The optical data}

Long-slit spectra were obtained with the Boller \& Chivens Cassegrain spectrograph at the ESO $1.52 \mathrm{~m}$ telescope (La Silla) during several runs in 1997 and 1998 as part of a key project during Brazilian time. We used the 
Table 1. Observed sample.

\begin{tabular}{|c|c|c|c|c|c|c|c|c|c|}
\hline $\begin{array}{c}\text { ESO-LV } \\
\text { Name } \\
(1)\end{array}$ & $\begin{array}{l}\text { Other } \\
\text { Name } \\
(2)\end{array}$ & $\begin{array}{c}\text { Coord. } \\
\text { J2000 } \\
(3)\end{array}$ & $\begin{array}{c}\text { Sample \& } \\
\text { Morph. } \\
\text { (4) }\end{array}$ & $\begin{array}{c}\text { Morph. } \\
\text { RC3 } \\
(5)\end{array}$ & $\begin{array}{l}N_{\mathrm{g}} \\
(6)\end{array}$ & $\begin{array}{c}r_{\mathrm{p}} \\
\mathrm{Mpc} \\
(7)\end{array}$ & $\begin{array}{l}B_{\mathrm{T}} \\
(8)\end{array}$ & $\begin{array}{c}F_{60 \mu \mathrm{m}} \\
\mathrm{Jy} \\
(9)\end{array}$ & $\begin{array}{c}F_{100 \mu \mathrm{m}} \\
\mathrm{Jy} \\
(10)\end{array}$ \\
\hline 5390050 & & $001710.1-191800$ & CS 5 & $\mathrm{SAB}(\mathrm{rs}) \mathrm{c} ?$ & & & 13.53 & 0.977 & 2.972 \\
\hline 3500140 & N101 & $002354.6-323209$ & CS 6 & $\mathrm{SAB}(\mathrm{rs}) \mathrm{c}$ & & & 13.37 & 0.549 & 1.754 \\
\hline 3520530 & N491 & $012120.3-340348$ & HDS 3 & $\mathrm{SB}(\mathrm{rs}) \mathrm{b}:$ & 3 & 0.18 & 13.21 & 2.843 & 8.632 \\
\hline 2960380 & & $013227.4-384040$ & CS 4 & $\mathrm{SAB}(\mathrm{rs}) \mathrm{c}$ & & & 13.99 & 0.516 & 1.779 \\
\hline 4780060 & & $020919.1-232454$ & CS 4 & $\mathrm{Sbc}$ & & & 13.22 & 3.543 & 9.112 \\
\hline 5450100 & N907 & $02 \quad 2301.7-204243$ & HDS 5 & SBdm? sp & 5 & 0.40 & 13.21 & 2.649 & 5.625 \\
\hline 5450110 & N908 & $022304.8-211403$ & HDS 5 & $\mathrm{SA}(\mathrm{s}) \mathrm{c}$ & & & 10.83 & 14.770 & 43.670 \\
\hline 3550260 & & $0232 \quad 17.5-350150$ & CS 4 & SB(s)bc: & & & 13.80 & 0.482 & 1.588 \\
\hline 3550300 & & $023736.4-325528$ & CS 4 & SB(rs:)bc: & & & 13.59 & 0.881 & 3.137 \\
\hline 0310050 & & $025806.0-742724$ & CS 3.5 & $\mathrm{SAB}(\mathrm{rs}) \mathrm{bc}$ & & & 14.07 & 1.043 & 3.887 \\
\hline 3570190 & N1310 & $032103.7-370558$ & HDS 5 & $\mathrm{SB}(\mathrm{rs}) \mathrm{cd}$ & 55 & 0.82 & 12.55 & 0.881 & 3.345 \\
\hline 5480070 & N1325 & $032425.6-213235$ & HDS 3.5 & $\mathrm{SA}(\mathrm{s}) \mathrm{bc}$ & 7 & 0.94 & 12.22 & 0.631 & 3.211 \\
\hline 5480310 & N1353 & $033203.0-204904$ & HDS 3 & $\mathrm{SA}(\mathrm{rs}) \mathrm{bc}$ & 7 & 0.94 & 12.40 & 2.420 & 8.786 \\
\hline 5480380 & I1953 & $033341.7-212845$ & HDS 6 & $\mathrm{SB}(\mathrm{rs}) \mathrm{d}$ & 7 & 0.94 & 12.24 & 8.470 & 11.128 \\
\hline 4190030 & & $03 \quad 42 \quad 11.2-275147$ & $\mathrm{CS} 4$ & $\left(\mathrm{R}^{\prime}\right) \mathrm{SAB}(\mathrm{rs}) \mathrm{c}$ & & & 13.60 & 1.334 & 3.361 \\
\hline 4820430 & N1459 & $034658.0-253111$ & $\mathrm{CS} 4$ & $\mathrm{SB}(\mathrm{s}) \mathrm{bc} ?$ & & & 13.62 & 0.572 & 2.657 \\
\hline 4200030 & & $040745.8-295130$ & CS 5 & $\mathrm{SA}(\mathrm{rs}) \mathrm{bc}$ & & & 13.52 & 0.704 & 2.172 \\
\hline 2010220 & & $0408 \quad 59.3-48 \quad 4342$ & CS 5 & $\mathrm{Sbc}$ & & & 14.73 & 0.356 & 1.466 \\
\hline 1570050 & N1536 & $041059.9-562848$ & HDS 5.5 & SB(s)c pec: & 46 & 1.30 & 13.15 & 0.475 & 1.649 \\
\hline 4840250 & N1591 & $042930.7-264244$ & CS 2 & $\mathrm{SB}(\mathrm{r}) \mathrm{ab}$ pec & & & 13.77 & 1.929 & 5.001 \\
\hline 1190060 & N1688 & $044823.8-594759$ & HDS 7.5 & $\mathrm{SB}(\mathrm{rs}) \mathrm{dm}$ & 14 & 0.85 & 12.57 & 2.683 & 6.677 \\
\hline 1190190 & N1703 & $045251.9-594433$ & HDS 5 & $\mathrm{SA}(\mathrm{s}) \mathrm{c}$ & 14 & 0.85 & 11.90 & 2.122 & 7.723 \\
\hline 3050140 & & $051234.1-395136$ & CS 5 & $\mathrm{SB}(\mathrm{s}) \mathrm{c}$ & & & 14.13 & 0.378 & 0.982 \\
\hline 2030180 & N1803 & $050526.6-493405$ & $\mathrm{CS} 4$ & Sbc: & & & 13.38 & 0.277 & 0.715 \\
\hline 1420500 & I4901 & $195423.1-584250$ & CS 5 & $\mathrm{SAB}(\mathrm{r}) \mathrm{c}$ & & & 12.29 & 1.778 & 6.518 \\
\hline 2340160 & & $202325.1-503243$ & HDS 5 & $\mathrm{SAB}(\mathrm{s}) \mathrm{bc}$ pec & 4 & 0.68 & 14.56 & 3.069 & 7.875 \\
\hline 2850080 & N6902 & $202427.7-433909$ & HDS 4 & $\mathrm{SA}(\mathrm{r}) \mathrm{b}$ & 4 & 0.31 & 11.64 & 0.826 & 3.924 \\
\hline 1060120 & I5038 & $204651.2-650100$ & CS 6 & $\left(\mathrm{R}^{\prime}:\right) \mathrm{SB}(\mathrm{s}) \mathrm{bc}$ & & & 14.13 & 0.723 & 2.460 \\
\hline 2350550 & & $2105 \quad 55.4-48 \quad 12 \quad 23$ & HDS 5 & $\left(\mathrm{R}^{\prime}\right) \mathrm{SAB}(\mathrm{rs}) \mathrm{bc}$ & 9 & 1.00 & 12.70 & 0.461 & 2.840 \\
\hline 2350570 & & $210621.8-481014$ & HDS 4 & Sbc: sp & 9 & 1.00 & 14.45 & 0.461 & 3.368 \\
\hline 2860820 & & $21 \quad 1545.4-422533$ & HDS 5 & $\mathrm{SAB}(\mathrm{s}) \mathrm{c}$ & 3 & 0.20 & 14.51 & 0.337 & 1.032 \\
\hline 2370020 & N7124 & $214805.7-503351$ & CS 4.5 & $\mathrm{SB}(\mathrm{rs}) \mathrm{c}$ & & & 13.10 & 0.791 & 3.411 \\
\hline 1890070 & N7140 & $2152 \quad 15.3 \quad-553410$ & CS 4 & $\left(\mathrm{R}_{2}^{\prime}\right) \mathrm{SB}(\mathrm{rs}) \mathrm{b}$ & & & 12.20 & 2.183 & 5.886 \\
\hline 2880260 & N7162 & $215939.0-43 \quad 18 \quad 12$ & $\operatorname{HDS} 5$ & $\left(\mathrm{R}^{\prime}\right) \mathrm{SA}(\mathrm{r}) \mathrm{bc}$ & 4 & 0.20 & 13.29 & 0.484 & 1.656 \\
\hline 5320090 & N7167 & $220030.9-243800$ & CS 5 & SB(s)c: & & & 13.22 & 1.314 & 3.588 \\
\hline 6010040 & & $220130.4-220415$ & CS 4.6 & $\mathrm{SB}(\mathrm{s}) \mathrm{c}:$ & & & 14.58 & 0.227 & 0.877 \\
\hline 1080130 & N7191 & $220651.3-643803$ & HDS 3.5 & $\mathrm{SAB}(\mathrm{rs}) \mathrm{c}$ & 5 & 0.48 & 13.80 & 0.570 & 2.061 \\
\hline 1080200 & $\mathrm{I} 5176$ & $221155.0-665046$ & CS 3.9 & $\mathrm{SAB}(\mathrm{s}) \mathrm{bc} ? \mathrm{sp}$ & & & 13.54 & 3.031 & 11.21 \\
\hline 1460090 & N7205 & $220834.4-572633$ & CS 5 & $\mathrm{SA}(\mathrm{s}) \mathrm{bc}$ & & & 11.55 & 8.861 & 25.960 \\
\hline 4050180 & N7267 & $222421.6-334138$ & CS 1 & $\left(\mathrm{R}_{1}^{\prime}\right) \mathrm{SB}(\mathrm{rs}) \mathrm{a}$ & & & 12.91 & 2.081 & 4.930 \\
\hline 4060250 & N7418 & $225636.0-370147$ & HDS 5 & $\mathrm{SAB}(\mathrm{rs}) \mathrm{cd}$ & 32 & 1.31 & 11.66 & 4.344 & 15.010 \\
\hline 4060330 & $\mathrm{I} 5270$ & $225754.7-355130$ & HDS 6 & $\mathrm{SB}(\mathrm{rs}) \mathrm{c}$ & 32 & 1.31 & 13.00 & 3.076 & 8.398 \\
\hline 4070140 & & $23 \quad 1739.7-344724$ & CS 5 & $\mathrm{SB}(\mathrm{s}) \mathrm{c} ?$ & & & 13.48 & 0.987 & 2.766 \\
\hline 3470340 & N7599 & $231921.1-421520$ & HDS 3 & $\mathrm{SB}(\mathrm{s}) \mathrm{c}$ & 32 & 1.31 & 12.08 & 5.408 & 21.750 \\
\hline 2400110 & & $233749.7-474342$ & HDS 4.8 & $\mathrm{Sb}$ & 3 & 0.18 & 13.20 & 0.956 & 5.612 \\
\hline 2400130 & & $233926.9-474627$ & HDS 3 & $\left(\mathrm{R}_{1}^{\prime}\right) \mathrm{SAB}(\mathrm{rs}) \mathrm{b}$ & 3 & 0.18 & 13.99 & 0.791 & 3.411 \\
\hline 4710200 & N7755 & $234551.8-303119$ & $\mathrm{CS} 4.5$ & $\mathrm{SB}(\mathrm{r}) \mathrm{bc}$ & & & 12.56 & 2.686 & 8.538 \\
\hline
\end{tabular}

Column 4: $\mathrm{CS}=$ control sample, HDS = high density sample; morphological types are: $1=\mathrm{Sa}, 2=\mathrm{Sa}-\mathrm{b}, 3=\mathrm{Sb}, 4=\mathrm{Sb}-\mathrm{c}$, $5=\mathrm{S} \ldots, 6=\mathrm{Sc}$, Sc-d, $7=\mathrm{S} . . / \mathrm{Irr}, 8=\mathrm{Sd}$. Column 6: $N_{\mathrm{g}}$ is the number of companions from Maia et al. (1989). Column 7: $r_{\mathrm{p}}$ is the mean pairwise separation from Maia et al. (1989).

Loral/Lesser CCD (No. 39) with $2048 \times 2048$ pixels $(1$ pixel $=15 \mu \mathrm{m})$ and grating No. 27 which has 600 lines $\mathrm{mm}^{-1}$ and gives a spectral coverage of $3600-7500 \AA$ and dispersion of $1.7 \AA \mathrm{pix}^{-1}$. The slit width was $3^{\prime \prime}$ and positioned along the major axis of the galaxies which corresponds to galactic sizes of $250 \mathrm{pc}$ for the closest galaxy in the sample and to $1 \mathrm{kpc}$ for the most distant galaxy in the sample. 
Table 2. Parameters of strong emission lines.

\begin{tabular}{|c|c|c|c|c|c|c|c|}
\hline $\begin{array}{l}\text { ESO-LV } \\
\text { name } \\
(1)\end{array}$ & $\begin{array}{l}\text { Sample \& } \\
\text { Morph. } \\
\quad(2)\end{array}$ & $\begin{array}{c}F(\mathrm{H} \beta) \times 10^{-15} \\
\operatorname{ergs~cm} \mathrm{cm}^{-2} \mathrm{~s}^{-1} \\
(3)\end{array}$ & 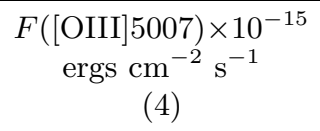 & $\begin{array}{c}F(\mathrm{H} \alpha) \times 10^{-15} \\
\operatorname{ergs~} \mathrm{cm}^{-2} \mathrm{~s}^{-1} \\
(5)\end{array}$ & 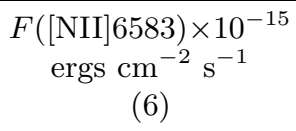 & $\begin{array}{c}E W(\mathrm{H} \alpha) \\
\AA \\
(7)^{\dagger}\end{array}$ & $\begin{array}{l}\text { Type of } \\
\text { Activity } \\
\text { (8) }\end{array}$ \\
\hline 0310050 & CS 3.5 & 4.0 & 0.3 & 15.0 & 8.2 & 8.2 & HII \\
\hline 1060120 & CS 6 & 10.2 & 1.5 & 38.0 & 18.0 & 12.4 & HII \\
\hline 1080130 & HDS 3.5 & 7.2 & 0.6 & 41.0 & 15.0 & 14.2 & HII \\
\hline 1190190 & HDS 5 & 5.7 & 1.3 & 12.0 & 6.7 & 5.5 & HII \\
\hline 1420500 & CS 5 & 10.0 & 2.8 & 20.0 & 16.0 & 3.0 & $\mathrm{~L}$ \\
\hline 1460090 & CS 5 & 21.0 & 5.1 & 60.0 & 31.0 & 5.6 & HII \\
\hline 1570050 & HDS 5.5 & 14.5 & 4.4 & 53.0 & 17.0 & 27.9 & HII \\
\hline 2010220 & CS 5 & 12.0 & 8.8 & 45.8 & 13.1 & 16.6 & HII \\
\hline 2030180 & CS 4 & 45.4 & 23.3 & 160 & 63.0 & 29.2 & HII \\
\hline 2340160 & HDS 5 & 11.6 & 8.0 & 40.0 & 15.0 & 29.6 & HII \\
\hline 2350550 & HDS 5 & 3.0 & 2.4 & 8.7 & 17.0 & & $\mathrm{~L}$ \\
\hline 2350570 & HDS 4 & 2.1 & 2.7 & 5.9 & 11.0 & 1.2 & $\mathrm{~L}$ \\
\hline 2370020 & CS 4.5 & 7.9 & 2.8 & 3.3 & 9.5 & 0.9 & $\mathrm{~L}$ \\
\hline 2400110 & HDS 4.8 & 12.0 & 0.9 & 16.6 & 18.5 & 1.9 & $\mathrm{~L}$ \\
\hline 2850080 & HDS 4 & 14.6 & 4.2 & 9.0 & 17.0 & 1.1 & $\mathrm{~L}$ \\
\hline 2860820 & HDS 5 & 8.0 & 0.9 & 27.0 & 11.0 & 14.6 & HII \\
\hline 2880260 & HDS 5 & 19.0 & 1.0 & 49.0 & 32.0 & 6.6 & $\mathrm{~L}$ \\
\hline 2960380 & CS 4 & 7.2 & 1.7 & 26.0 & 10.0 & 16.5 & HII \\
\hline 3050140 & CS 5 & 1.8 & 0.4 & 6.7 & 3.8 & 8.7 & HII \\
\hline 3500140 & CS 6 & 8.7 & 2.6 & 33.0 & 14.0 & 13.3 & HII \\
\hline 3550300 & CS 4 & 12.2 & 2.6 & 22.2 & 18.0 & 3.7 & $\mathrm{~L}$ \\
\hline 3570190 & HDS 5 & 13.4 & 8.4 & 50.0 & 22.0 & 14.9 & HII \\
\hline 4060330 & HDS 6 & 43.0 & 21.0 & 180 & 66.0 & 27.4 & HII \\
\hline 4070140 & CS 5 & 53.2 & 51.0 & 190 & 58.0 & 40.6 & HII \\
\hline 4190030 & $\mathrm{CS} 4$ & 5.1 & 1.6 & 22.0 & 7.8 & 24.2 & HII \\
\hline 4200030 & CS 5 & 15.2 & 4.7 & 48.0 & 18.0 & 13.3 & HII \\
\hline 4710200 & CS 4.5 & 24.3 & 6.5 & 98.0 & 47.0 & 10.3 & HII \\
\hline 4780060 & CS 4 & 23.1 & 6.0 & 63.0 & 26.0 & 13.7 & HII \\
\hline 4820430 & CS 4 & 6.9 & 2.6 & 17.0 & 9.4 & 6.7 & HII \\
\hline 5320090 & CS 5 & 16.8 & 7.3 & 51.0 & 21.0 & 13.4 & HII \\
\hline 5390050 & CS 5 & 16.7 & 9.2 & 64.0 & 28.0 & 20.0 & HII \\
\hline 5450100 & HDS 5 & 23.4 & 24.7 & 95.0 & 26.0 & 30.0 & HII \\
\hline 5480310 & HDS 3 & 16.0 & 1.0 & 53.0 & 35.0 & 4.2 & $\mathrm{~L}$ \\
\hline 5480380 & HDS 6 & 5.6 & 1.4 & 24.0 & 9.6 & 28.8 & HII \\
\hline 6010040 & CS 4.6 & 8.6 & 1.0 & 30.0 & 14.0 & 6.6 & HII \\
\hline
\end{tabular}

Column 2: $\mathrm{CS}=$ control sample, $\mathrm{HDS}=$ high density sample; morphological types are: $1=\mathrm{Sa}, 2=\mathrm{Sa}-\mathrm{b}, 3=\mathrm{Sb}, 4=\mathrm{Sb}-\mathrm{c}$, $5=\mathrm{S} \ldots, 6=\mathrm{Sc}$, Sc-d, $7=\mathrm{S} . . / \mathrm{Irr}, 8=\mathrm{Sd}$. Column 8 : HII = activity typical of HII regions, L = activity typical of LINERs.

$\dagger$ Only $E W(\mathrm{H} \alpha)$ is corrected for internal reddening.

Spectrophotometric standard stars were observed close to zenith several times during the night with a slit width of $5^{\prime \prime}$. A He-Ar lamp was observed after every exposure and used for wavelength calibration. Typical exposure times were $2 \times 20-30 \mathrm{~min}$ for galaxies and 5-10 min for stars.

Standard data reduction, including bias and flat-field correction, was performed using IRAF. One dimensional spectra were extracted from each galaxy integrated along the slit length. We corrected for Galactic extinction using the Cardelli et al. (1989) extinction curve and $E(B-V)$ from NED. All spectra are flux calibrated and corrected for Doppler shift which was calculated using a crosscorrelation technique.

Starlight subtraction was particularly critical in weak lines such as $\mathrm{H} \beta$. The starlight contribution was removed using the technique of McCall et al. (1985, see also StorchiBergmann et al. 1994). Taking into account that in the typical stellar population the equivalent width of $\mathrm{H} \beta$ in absorption is of the order of $1.5 \AA$, we corrected for this effect by adding a factor of 1.5 times the continuum flux around $\mathrm{H} \beta$ to the emission line flux. When no emission line was clearly visible we adopted a theoretical ratio, $\mathrm{H} \alpha / \mathrm{H} \beta=2.86$ (Ho et al. 1997). In this case, the value of $\mathrm{H} \beta$ is an upper limit. Therefore, higher ratios of $\mathrm{H} \alpha / \mathrm{H} \beta$ can also be expected. We have investigated whether a higher ratio would influence our results by adopting ratios typical of AGNs $(\mathrm{H} \alpha / \mathrm{H} \beta=3.1)$. We found no significant difference given the uncertainties in the continuum determination.

We tested a second method of starlight subtraction using templates of old stellar populations from Bica (1988). 

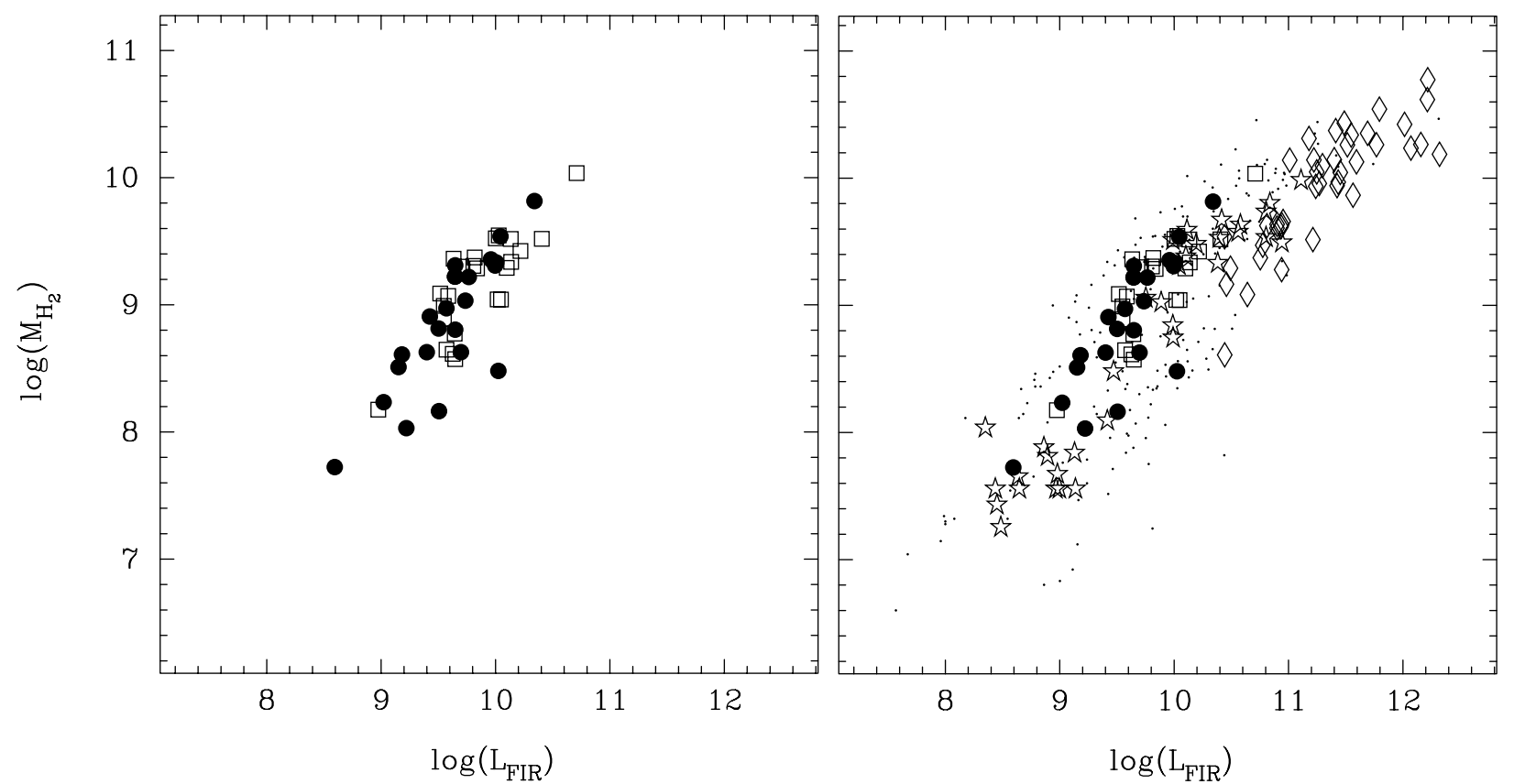

Fig. 1. Left panel a): total molecular gas as a function of FIR luminosity. The CS is marked by open squares and HDS by filled circles. Right panel b): the same as in the left panel. Ultraluminous infrared galaxies (Sanders et al. 1991) are marked by open diamonds, normal spiral galaxies from Young et al. (1989) and Braine et al. (1993) are marked by dots, cluster galaxies (Coma and Fornax) from Casoli et al. (1991) and Horellou et al. (1995) are marked by open stars. Luminosity is in $L_{\odot}$ and mass is in $M_{\odot}$.

We subtracted our spectra from the templates and then measured the fluxes. Both methods gave similar results given the accuracy of the measurements, dominated by the uncertainty in the continuum determination (Cid Fernandes et al. 1998).

We measured the integrated fluxes and equivalent widths of the emission lines $\mathrm{H} \beta$, [OIII] $\lambda 5007$, [NII] $\lambda 6548$, $\mathrm{H} \alpha$, [NII] $\lambda 6583$, [SII] $\lambda 6716,6731$ for 35 galaxies with good signal-to-noise spectra. Internal reddening was estimated from the Cardelli et al. (1989) extinction curve and $\mathrm{H} \alpha / \mathrm{H} \beta$ ratios. $\mathrm{H} \alpha$ equivalent width was measured after internal reddening correction, following the same procedure as in Ho et al. (1997).

The type of activity was classified by measuring line-intensity ratios $(\log ([\mathrm{OIII}] \quad \lambda \quad 5007 / \mathrm{H} \beta)$ and $\log ([\mathrm{NII}] \lambda 6583 / \mathrm{H} \alpha))$ and applying standard diagnostic diagrams (Baldwin et al. 1981; Veilleux \& Osterbrock 1987). In Paper II we show the diagnostic diagram used to classify the type of activity.

Table 2 lists the emission line parameters as follows. Column 1: designation in the ESO-Uppsala cata$\log$ (LV89); Col. 2: type of sample (control sample = CS and high density sample $=$ HDS) and morphological type (LV89) $1=\mathrm{Sa}, 2=\mathrm{Sa}-\mathrm{b}, 3=\mathrm{Sb}, 4=\mathrm{Sb}-\mathrm{c}, 5=\mathrm{S} \ldots$, $6=\mathrm{Sc}, \mathrm{Sc}-\mathrm{d}, 7=\mathrm{S} . . / \mathrm{Irr}, 8=\mathrm{Sd}$; Col. $3: \mathrm{H} \beta$ flux; Col. 4 : [OIII] $\lambda 5007$ flux; Col. 5: H $\alpha$ flux; Col. 6: [NII] 6583 flux; Col. 7: $\mathrm{H} \alpha$ equivalent width in $\AA$, and Col. 8: type of activity ( $\mathrm{L}=$ LINERS, HII $=$ HII region $)$.

In Appendix A (only available in electronic form) we show the optical spectra of 35 galaxies of our sample. We also included in the Appendix the CO spectra described below and images from The Digitized Sky Surveys ${ }^{1}$ which allows direct inspection of the galaxies morphology.

\section{The CO data}

Millimetric observations were carried out at the SwedishESO (SEST) $15 \mathrm{~m}$ radiotelescope at La Silla in October 1996 and September 1998 during good weather conditions. In the first run we used the SESIS 100 receiver with a $1 \mathrm{GHz}$ bandwidth at $115 \mathrm{GHz}\left({ }^{12} \mathrm{CO}(1-0)\right)$. Typical system temperatures were $\sim 250 \mathrm{~K}$ (in the $T_{\mathrm{A}}^{*}$ scale) at the elevation of the sources and typical zenith opacities between 0.1-0.2. During the second run we used the IRAM 115 and IRAM 230 receivers with $500 \mathrm{MHz}$ and $1 \mathrm{GHz}$ bandwith, at $115 \mathrm{GHz}\left({ }^{12} \mathrm{CO}(1-0)\right)$ and $230 \mathrm{GHz}$ $\left({ }^{12} \mathrm{CO}(2-1)\right)$, respectively. The half power beamwidth of the SEST at $115 \mathrm{GHz}$ is $45^{\prime \prime}$ and $23^{\prime \prime}$ at $230 \mathrm{GHz}$.

All galaxies were observed at the central optical coordinate. Integration times were $2-3$ hours depending on the signal-to-noise achieved. The pointing was regularly checked on nearby $\mathrm{SiO}$ masers. The pointing uncertainties were of the order of $5^{\prime \prime}$. CO emission was detected in 47 galaxies and had low signal-to-noise detection in only 5 galaxies, ESO-LV1080110 (HDS), ESO-LV1880170 (CS), ESO-LV2850050 (HDS), ESO-LV3550300 (CS), and ESOLV6050070 (CS). We have not included these galaxies in our analysis.

\footnotetext{
1 The Digitized Sky Surveys were produced at the Space Telescope Science Institute under US Government grant NAG W-2166.
} 
Table 3. CO data.

\begin{tabular}{|c|c|c|c|c|c|c|c|c|}
\hline $\begin{array}{c}\text { ESO-LV } \\
\text { name } \\
(1)\end{array}$ & $\begin{array}{l}\text { Sample \& } \\
\text { Morph. } \\
\text { (2) }\end{array}$ & $\begin{array}{c}V_{\mathrm{CO}} \\
\mathrm{km} \mathrm{s}^{-1} \\
(3)\end{array}$ & $\begin{array}{c}\Delta V_{\mathrm{CO}} \\
\mathrm{km} \mathrm{s}^{-1} \\
(4)\end{array}$ & $\begin{array}{c}\log L_{\mathrm{B}} \\
L_{\odot} \\
(5)\end{array}$ & $\begin{array}{c}L_{\mathrm{FIR}} \times 10^{9} \\
L_{\odot} \\
(6)\end{array}$ & $\begin{array}{c}I_{\mathrm{CO}(1-0)} \\
\mathrm{K} \mathrm{km} \mathrm{s}^{-1} \\
\quad(7)\end{array}$ & $\begin{array}{c}M_{\mathrm{H}_{2}} \times 10^{9} \\
M_{\odot} \\
(8)\end{array}$ & $\begin{array}{l}I_{\mathrm{CO}(2-1)} \\
\mathrm{K} \mathrm{km} \mathrm{s}^{-1}\end{array}$ \\
\hline 0310050 & CS 3.5 & 4714 & 287 & 10.11 & $13.60 \pm 0.39$ & $3.62 \pm 0.26$ & $3.30 \pm 0.23$ & $3.64 \pm 0.22$ \\
\hline 1060120 & CS 6 & 4154 & 180 & 9.97 & $6.90 \pm 0.39$ & $2.75 \pm 0.28$ & $1.93 \pm 0.19$ & \\
\hline 1080130 & HDS 3.5 & 2941 & 135 & 9.78 & $2.67 \pm 0.15$ & $2.43 \pm 0.21$ & $0.81 \pm 0.07$ & \\
\hline 1080200 & CS 3.9 & 1720 & 183 & 9.37 & $4.45 \pm 0.20$ & $6.28 \pm 0.25$ & $0.65 \pm 0.03$ & $3.63 \pm 0.18$ \\
\hline 1190060 & HDS 7.5 & 1256 & 43 & 9.48 & $1.66 \pm 0.05$ & $1.99 \pm 0.14$ & $0.11 \pm 0.01$ & \\
\hline 1190190 & HDS 5 & 1527 & 33 & 9.94 & $2.52 \pm 0.07$ & $5.06 \pm 0.19$ & $0.42 \pm 0.02$ & \\
\hline 1420500 & CS 5 & 2135 & 165 & 10.10 & $4.40 \pm 0.09$ & $3.40 \pm 0.25$ & $0.59 \pm 0.04$ & \\
\hline 1460090 & CS 5 & 1652 & 183 & 10.13 & $10.40 \pm 0.34$ & $11.68 \pm 0.66$ & $1.10 \pm 0.06$ & \\
\hline 1570050 & HDS 5.5 & 1311 & 40 & 9.30 & $0.39 \pm 0.02$ & $1.66 \pm 0.06$ & $0.10 \pm 0.01$ & $0.88 \pm 0.09$ \\
\hline 1890070 & CS 4.0 & 3006 & 169 & 10.44 & $9.15 \pm 0.36$ & $4.22 \pm 0.26$ & $1.48 \pm 0.09$ & \\
\hline 2010220 & CS 5 & 3990 & 188 & 9.70 & $3.53 \pm 0.23$ & $1.50 \pm 0.11$ & $0.98 \pm 0.07$ & $1.30 \pm 0.11$ \\
\hline 2030180 & $\mathrm{CS} 4$ & 4123 & 157 & 10.27 & $25.39 \pm 1.17$ & $4.66 \pm 0.23$ & $3.30 \pm 0.16$ & $5.04 \pm 0.14$ \\
\hline 2340160 & HDS 5 & 5218 & 10 & 10.01 & $3.72 \pm 0.50$ & $0.82 \pm 0.05$ & $0.94 \pm 0.06$ & $0.45 \pm 0.12$ \\
\hline 2350550 & HDS 5 & 5098 & 70 & 10.73 & $9.92 \pm 1.11$ & $1.88 \pm 0.11$ & $2.04 \pm 0.12$ & $1.34 \pm 0.11$ \\
\hline 2350570 & HDS 4 & 5069 & 248 & 10.03 & $11.08 \pm 1.30$ & $3.22 \pm 0.12$ & $3.45 \pm 0.13$ & $3.56 \pm 0.22$ \\
\hline 2370020 & CS 4.5 & 5214 & 236 & 10.58 & $13.72 \pm 0.70$ & $4.42 \pm 0.16$ & $4.90 \pm 0.18$ & $1.96 \pm 0.11$ \\
\hline 2400110 & HDS 4.8 & 2890 & 278 & 10.00 & $5.81 \pm 0.31$ & $5.20 \pm 0.17$ & $1.65 \pm 0.05$ & \\
\hline 2400130 & HDS 3 & 3284 & 50 & 9.80 & $5.17 \pm 0.31$ & $2.58 \pm 0.15$ & $1.08 \pm 0.06$ & \\
\hline 2850080 & HDS 4 & 2838 & 132 & 10.63 & $4.45 \pm 0.23$ & $1.97 \pm 0.21$ & $0.64 \pm 0.07$ & \\
\hline 2860820 & HDS 5 & 4958 & 134 & 9.98 & $4.42 \pm 0.51$ & $1.62 \pm 0.09$ & $1.66 \pm 0.09$ & $1.68 \pm 0.08$ \\
\hline 2880260 & HDS 5 & 2383 & 80 & 9.79 & $1.42 \pm 0.10$ & $1.51 \pm 0.11$ & $0.32 \pm 0.02$ & \\
\hline 2960380 & CS 4 & 3645 & 51 & 9.90 & $3.73 \pm 0.33$ & $0.84 \pm 0.12$ & $0.44 \pm 0.06$ & \\
\hline 3050140 & CS 5 & 4761 & 450 & 10.11 & $4.31 \pm 0.55$ & $2.38 \pm 0.10$ & $2.31 \pm 0.10$ & $1.21 \pm 0.08$ \\
\hline 3470340 & HDS 3 & 1671 & 117 & 9.92 & $7.85 \pm 0.79$ & $23.46^{\dagger} \pm 0.62$ & $2.27 \pm 0.06$ & \\
\hline 3500140 & CS 6 & 3400 & 35 & 10.09 & $3.30 \pm 0.25$ & $2.68 \pm 0.09$ & $1.23 \pm 0.04$ & \\
\hline 3520530 & HDS 3 & 3874 & 260 & 10.27 & $21.89 \pm 0.93$ & $10.85 \pm 0.52$ & $6.55 \pm 0.32$ & \\
\hline 3550260 & CS 4 & 1985 & 105 & 9.42 & $0.95 \pm 0.07$ & $1.02 \pm 0.13$ & $0.15 \pm 0.02$ & \\
\hline 3550300 & $\mathrm{CS} 4$ & 4448 & 336 & 10.25 & $10.05 \pm 0.43$ & $4.08 \pm 0.44$ & $3.33 \pm 0.36$ & \\
\hline 3570190 & HDS 5 & 1789 & 66 & 9.83 & $1.52 \pm 0.06$ & $3.40 \pm 0.28$ & $0.41 \pm 0.03$ & \\
\hline 4050180 & CS 1 & 3375 & 124 & 10.27 & $10.67 \pm 0.68$ & $7.70 \pm 0.37$ & $3.52 \pm 0.17$ & \\
\hline 4060250 & HDS 5 & 1470 & 83 & 9.98 & $4.42 \pm 0.20$ & $27.44^{\ddagger} \pm 0.74$ & $2.04 \pm 0.05$ & \\
\hline 4060330 & HDS 6 & 1922 & 110 & 9.71 & $5.01 \pm 0.21$ & $3.15 \pm 0.17$ & $0.42 \pm 0.02$ & \\
\hline 4070140 & CS 5 & 2761 & 129 & 9.85 & $3.54 \pm 0.23$ & $2.64 \pm 0.14$ & $0.78 \pm 0.04$ & \\
\hline 4190030 & $\mathrm{CS} 4$ & 4146 & 83 & 10.20 & $11.19 \pm 0.36$ & $1.52 \pm 0.13$ & $1.10 \pm 0.09$ & \\
\hline 4200030 & CS 5 & 4093 & 163 & 10.22 & $6.41 \pm 0.41$ & $2.86 \pm 0.20$ & $2.02 \pm 0.14$ & \\
\hline 4710200 & CS 4.5 & 3017 & 160 & 10.30 & $12.49 \pm 0.53$ & $5.46 \pm 0.36$ & $1.95 \pm 0.13$ & \\
\hline 4780060 & CS 4 & 5401 & 164 & 10.58 & $51.12 \pm 2.91$ & $8.79 \pm 0.37$ & $10.86 \pm 0.46$ & $15.16 \pm 0.33$ \\
\hline 4820430 & CS 4 & 4073 & 85 & 10.17 & $6.57 \pm 0.33$ & $3.36 \pm 0.30$ & $2.35 \pm 0.21$ & \\
\hline 4840250 & $\mathrm{CS} 2$ & 4128 & 191 & 10.13 & $16.54 \pm 0.64$ & $3.63 \pm 0.31$ & $2.65 \pm 0.22$ & \\
\hline 5320090 & CS 5 & 2582 & 83 & 9.91 & $4.22 \pm 0.20$ & $1.54 \pm 0.17$ & $0.41 \pm 0.05$ & \\
\hline 5390050 & CS 5 & 3158 & 256 & 9.98 & $5.03 \pm 0.29$ & $4.95 \pm 0.33$ & $1.99 \pm 0.13$ & \\
\hline 5450100 & HDS 5 & 1715 & 21 & 9.55 & $3.21 \pm 0.14$ & $1.29 \pm 0.09$ & $0.15 \pm 0.01$ & $2.67 \pm 0.13$ \\
\hline 5450110 & HDS 5 & 1456 & 168 & 10.35 & $14.78 \pm 0.72$ & $27.01 \pm 0.92$ & $2.15 \pm 0.07$ & \\
\hline 5480070 & HDS 3.5 & 1557 & 17 & 9.87 & $1.05 \pm 0.05$ & $1.79 \pm 0.11$ & $0.17 \pm 0.01$ & \\
\hline 5480310 & HDS 3 & 1531 & 108 & 9.79 & $3.17 \pm 0.13$ & $6.99 \pm 0.32$ & $0.65 \pm 0.03$ & \\
\hline 5480380 & HDS 6 & 1874 & 86 & 10.03 & $10.56 \pm 0.31$ & $2.14 \pm 0.15$ & $0.30 \pm 0.02$ & \\
\hline 6010040 & CS 4.6 & 5219 & 103 & 10.01 & $3.85 \pm 0.56$ & $1.01 \pm 0.08$ & $1.17 \pm 0.09$ & $0.78 \pm 0.04$ \\
\hline
\end{tabular}

$\dagger$ added $\mathrm{CO}(1-0)$ intensities of 5 points (map);

$\ddagger$ added $\mathrm{CO}(1-0)$ intenstities of 7 points (map).

Column 2: $\mathrm{CS}=$ control sample, HDS $=$ high density sample; morphological types are: $1=\mathrm{Sa}, 2=\mathrm{Sa}-\mathrm{b}, 3=\mathrm{Sb}, 4=\mathrm{Sb}-\mathrm{c}$, $5=\mathrm{S} . . ., 6=\mathrm{Sc}, \mathrm{Sc}-\mathrm{d}, 7=\mathrm{S} . . / \mathrm{Irr}, 8=\mathrm{Sd}$.

Two galaxies, ESO-LV3470340 and ESO-LV4060250, were considerably larger than the SEST beam and were half of a beamwidth $\left(23^{\prime \prime}\right)$. In Appendix A we show each 

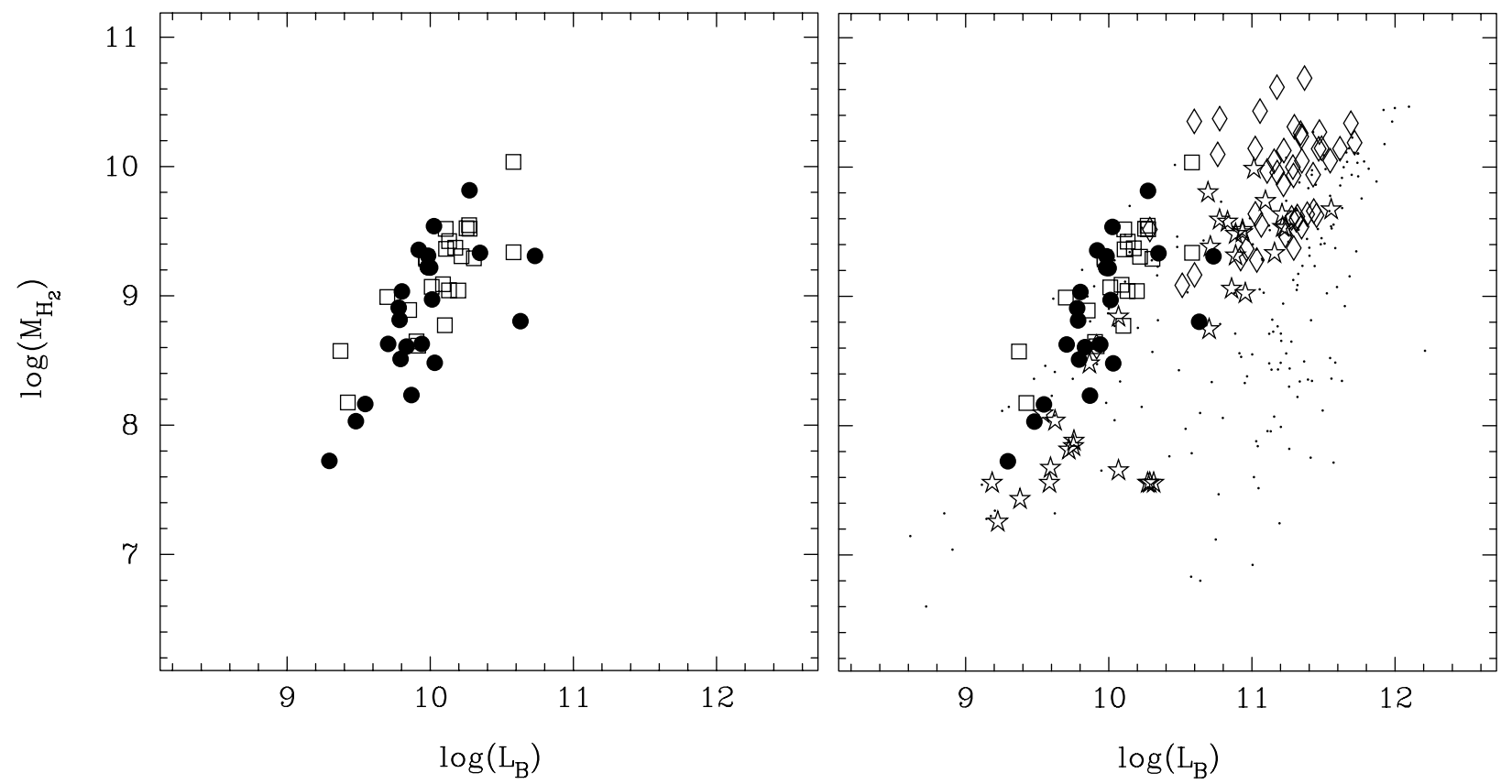

Fig. 2. Left panel a): total molecular gas as a function of blue luminosity. Right panel b): the same as in the left panel. Additional samples of ultraluminous infrared galaxies, galaxies in clusters, and spiral galaxies are included. Symbols are the same as in Fig. 1. Luminosity is in $L_{\odot}$ and mass is in $M_{\odot}$.

position along the major axis of the galaxy and give their spectra. We have added the intensities at each position in order to obtain the total $\mathrm{CO}$ intensity of each galaxy.

The CO spectra were reduced with the CLASS package (Forveille et al. 1990). We have binned the spectra with a boxcar function. Spectra were corrected for first order baseline in most of the cases or third order in a few obvious cases where first order did not give a good fit to the data. CO intensities were calculated by using the mainbeam efficiency, $\eta_{\mathrm{mb}}$, values of 0.7 and 0.5 for $115 \mathrm{GHz}$ and $230 \mathrm{GHz}$, respectively. We estimated the $1 \sigma$ uncertainty in the integrated line intensity taking into account the channel-to-channel noise (rms), the width of the emission profile $(\Delta V)$ and the number of channels $(N)$ that the emission profile covers (error $=\operatorname{rms} \times \Delta V \times N^{-1 / 2}$ ).

Table 3 lists the CO data as follows. Column 1: designation in the ESO-Uppsala catalog (LV89); Col. 2: type of sample (control sample $=\mathrm{CS}$ and high density sample $=$ HDS $)$ and morphological type (LV89) $1=\mathrm{Sa}$, $2=\mathrm{Sa}-\mathrm{b}, 3=\mathrm{Sb}, 4=\mathrm{Sb}-\mathrm{c}, 5=\mathrm{S} \ldots, 6=\mathrm{Sc}, \mathrm{Sc}-\mathrm{d}$, $7=\mathrm{S} . . / \mathrm{Irr}, 8=\mathrm{Sd}$; Col. 3: velocity derived from central $\mathrm{CO}(1-0)$ profiles in $\mathrm{km} \mathrm{s}^{-1}$; Col. 4: the width of the emission profile in $\mathrm{km} \mathrm{s}^{-1}$; Col. 5 : blue luminosity in $L_{\odot}$ derived from $B_{\mathrm{T}}$ magnitude (errors in $L_{\mathrm{B}}$ are within $10 \%$ when the magnitude estimates in the RC3 have errors of $0.1 \mathrm{mag}$ ); Col. 6: far-infrared luminosity in $L_{\odot}$ calculated as described in the next section; Col. 7: CO intensity in the line $J=(1-0)$ in $\mathrm{K} \mathrm{km} \mathrm{s}^{-1}$ and errors; Col. 8: $\mathrm{H}_{2}$ masses and errors in $M_{\odot}$ estimated from the velocity integrated $\mathrm{CO}(1-0)$ emission as described in the next section, and Col. 9: CO intensity in the line $J=(2-1)$ in $\mathrm{K} \mathrm{km} \mathrm{s}^{-1}$. Distances were corrected for the Virgocentric
Table 4. CO data from the literature.

\begin{tabular}{clcl}
\hline $\begin{array}{c}\text { ESO-LV } \\
\text { name }\end{array}$ & Sample & $\begin{array}{c}I_{\mathrm{CO}(1-0)} \\
\mathrm{K} \mathrm{kms}^{-1}\end{array}$ & \multicolumn{1}{c}{ References } \\
\hline 1060120 & CS & 2.2 & Combes et al. $(1994)^{\dagger}$ \\
1570050 & HDS & $<1.2$ & Horellou \& Booth $(1997)^{\dagger}$ \\
3570190 & HDS & $<0.6$ & Horellou \& Booth $(1997)^{\dagger}$ \\
4780060 & CS & $5.4 \pm 1.8$ & Andreani et al. $(1995)^{\dagger}$ \\
4840250 & CS & $3.5 \pm 0.7$ & Andreani et al. $(1995)^{\dagger}$ \\
5450110 & HDS & $12.2 \pm 0.8$ & Elfhag et al. $(1996)^{\ddagger}$ \\
5480380 & HDS & 4.4 & Combes et al. $(1994)^{\dagger}$ \\
\hline
\end{tabular}

${ }^{\dagger}$ Using SEST; ${ }^{\ddagger}$ using Onsala $20 \mathrm{~m}$.

flow according to model 3.1 in Aaronson et al. (1982). Hubble constant value of $75 \mathrm{~km} \mathrm{~s}^{-1} \mathrm{Mpc}^{-1}$ was adopted in all calculations.

Table 4 lists the CO intensity in the line $J=(1-0)$ available in the literature for 7 galaxies (4 in the HDS and 3 in the CS). The differences between the fluxes we have measured and the ones obtained previously are due to (i) different sizes of the beam (Elfhag et al. 1996); (ii) baseline adjustments (Combes et al. 1994; Andreani et al. 1995), or short integration time (Horellou \& Booth 1997).

\section{General properties}

The FIR emission together with the molecular gas provide unique information in terms of fuel and star formation. 
Table 5. Average values.

\begin{tabular}{lcccc}
\hline \multicolumn{1}{c}{ Sample } & $\log L_{\mathrm{B}}$ & $\log L_{\mathrm{FIR}}$ & $\log M_{\mathrm{H}_{2}}$ & $E W(\mathrm{H} \alpha)^{\dagger}$ \\
& $L_{\odot}$ & $L_{\odot}$ & $M_{\odot}$ & $\AA$ \\
\hline HDS mean & $9.94 \pm 0.33$ & $9.59 \pm 0.40$ & $8.86 \pm 0.39$ & $15.9 \pm 11.3$ \\
HDS median & $9.94 \pm 0.12$ & $9.65 \pm 0.28$ & $8.91 \pm 0.40$ & $14.2 \pm 11.2$ \\
CS mean & $10.08 \pm 0.29$ & $9.85 \pm 0.35$ & $9.18 \pm 0.39$ & $8.7 \pm 3.4$ \\
CS median & $10.11 \pm 0.14$ & $9.82 \pm 0.21$ & $9.29 \pm 0.22$ & $8.2 \pm 3.3$ \\
\hline
\end{tabular}

$\dagger$ Without LINERs.
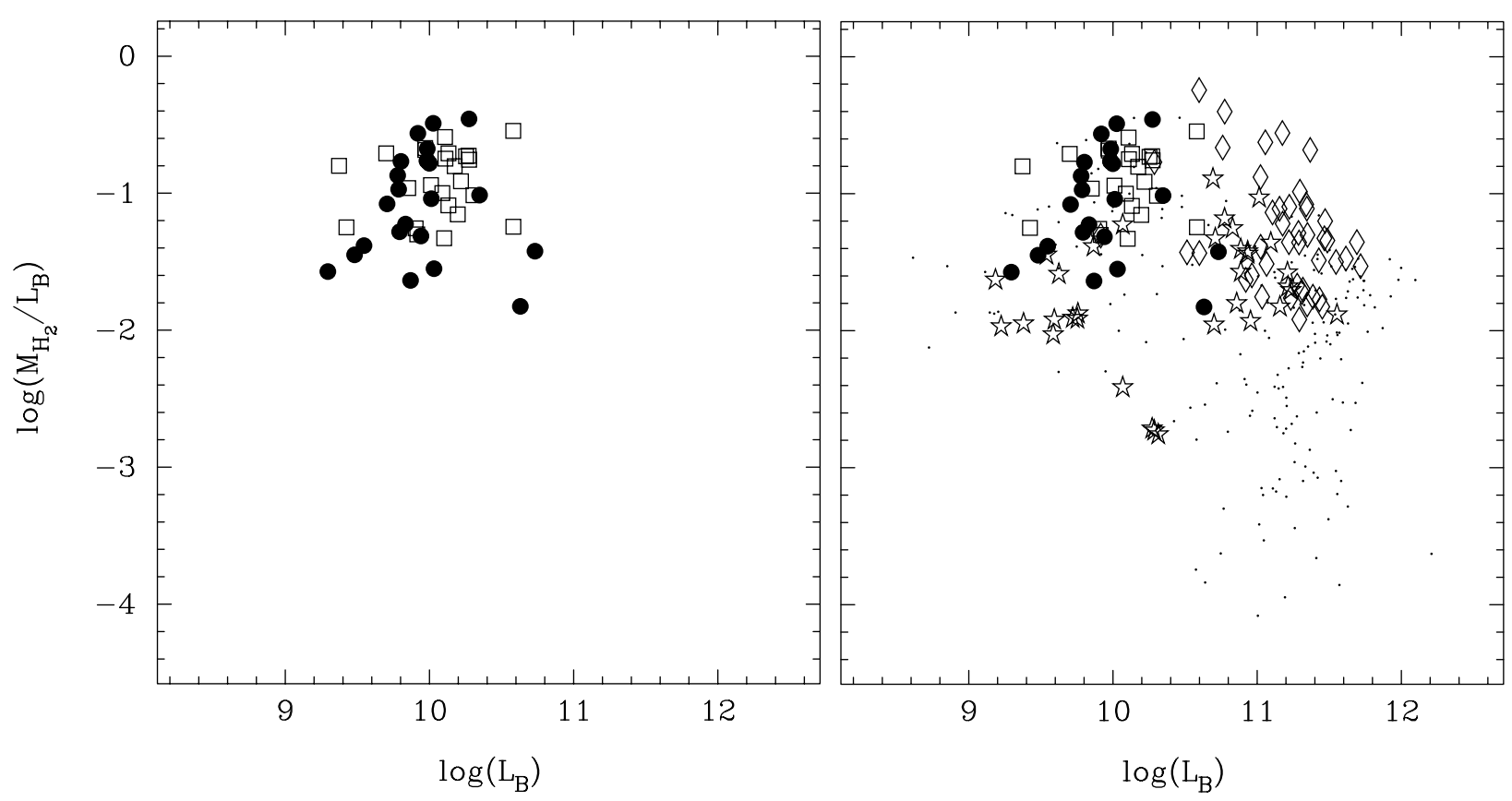

Fig. 3. Left panel a): total molecular gas normalized by the blue luminosity as a function of blue luminosity. Right panel b): the same as in the left panel. Additional samples of ultraluminous infrared galaxies, galaxies in clusters, and spiral galaxies are included. Symbols are the same as in Fig. 1. Luminosity is in $L_{\odot}$ and mass is in $M_{\odot}$.

The FIR luminosity was calculated using the relation (Lonsdale \& Helou 1985)

$$
L_{\mathrm{FIR}}=5.9 \times 10^{5} D^{2}\left(2.58 \times F_{60}+F_{100}\right)
$$

where $F_{60}$ and $F_{100}$ are fluxes in Jy at 60 and $100 \mu \mathrm{m}$ detected by IRAS and $D$ is the distance in Mpc corrected for the Virgo infall.

$\mathrm{H}_{2}$ masses were estimated from the velocity integrated $\mathrm{CO}(1-0)$ emission, using a $N_{\mathrm{H}_{2}} / I_{\mathrm{CO}}$ conversion ratio of $3 \times 10^{20} \mathrm{~cm}^{-2}\left(\mathrm{~K} \mathrm{~km} \mathrm{~s}^{-1}\right)$.

We are assuming that the conversion factor is the same in all galaxies in our sample. This assumption is reasonable since our sample do not contain any later-type systems (Sd, Sm, Ir) which, despite the ongoing star formation, show weak CO emission (e.g. Rubio et al. 1991).

Average and median values of $L_{\mathrm{B}}, L_{\mathrm{FIR}}, M_{\mathrm{H}_{2}}$, and $\mathrm{H} \alpha$ equivalent width are presented in Table 5 .

Figures 1a and 2a show the total amount of molecular gas as a function of FIR and blue luminosities. Figure 1a confirms the known correlation between $L_{\mathrm{FIR}}$ and the $\mathrm{H}_{2}$ masses (correlation coefficient $=0.80$ and 0.84 for the
HDS and CS, respectively). From Fig. 2a we verify that galaxies in the CS are on average more luminous than those in the HDS (a distance bias in our subsample). In order to eliminate this effect, $\mathrm{CO}$ intensities were normalized by the blue luminosity, $L_{\mathrm{B}}$, in the analysis presented in Paper II. Given our morphological selection criteria, we assumed that the mass $/ L_{\mathrm{B}}$ ratio is approximately the same for our galaxies (Roberts \& Haynes 1994) and $L_{\mathrm{B}}$ is thus a measure of the total mass.

We have plotted the $M_{\mathrm{H}_{2}} / L_{\mathrm{B}}$ as a function of $L_{\mathrm{B}}$ (Fig. 3a) in order to compare whether the bias in blue luminosity present in our subsample may cause a bias in our analysis. The correlation found for HDS and CS is very similar (correlation coefficient $=-0.03$ and 0.06 for the HDS and CS, respectively) suggesting no evident bias. We have compared our sample properties with samples observed by others, such as normal spiral galaxies (Young et al. 1989; Braine et al. 1993), the ultraluminous FIR galaxies (Sanders et al. 1991), and galaxies in the Coma and Fornax clusters (Casoli et al. 1991; Horellou et al. 1995). As it is shown in Figs. 1b, 2b, and 3b the 47 spiral 

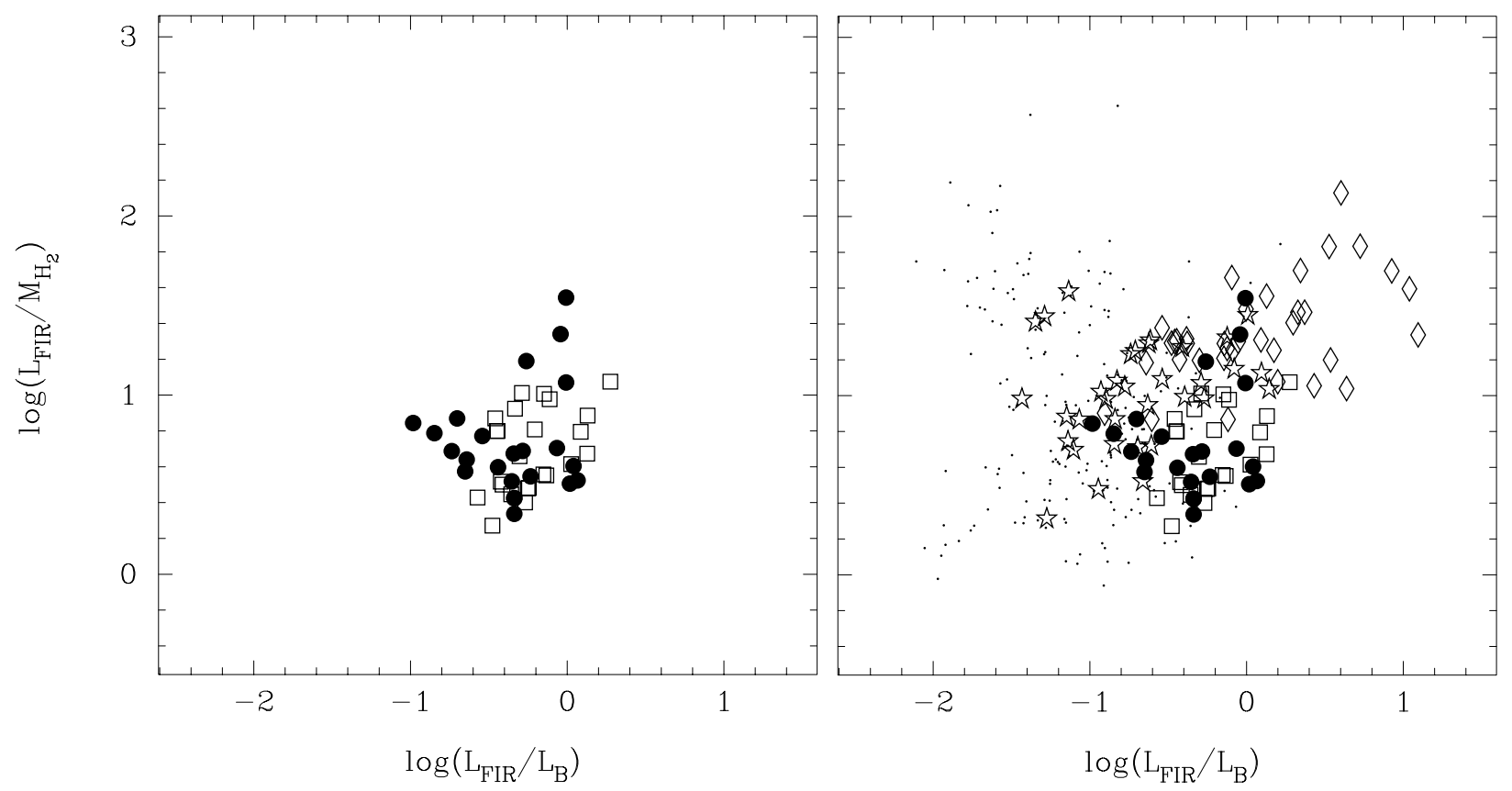

Fig. 4. Left panel a): FIR luminosity normalized by the total molecular gas as a function of the FIR luminosity normalized by the blue luminosity. Right panel b): the same as in the left panel. Additional samples of ultraluminous infrared galaxies, galaxies in clusters, and spiral galaxies are included. Symbols are the same as in Fig. 1. Luminosity is in $L_{\odot}$ and mass is in $M_{\odot}$.

galaxies of our sample (HDS and CS) have correlations between global parameters which are similar to those in other samples. The ultraluminous FIR galaxies (Sanders \& Mirabel 1996), as expected, are overall brighter and more massive than our subsample. The other samples include spirals of all types which explains the large dispersion found in luminosities and masses.

As previously mentioned, only intermediate Hubble types (Sb, Sbc, and $\mathrm{Sc}$ ) were selected in order to avoid any bias due to the correlation between general properties and morphology. However, even in this sample the uncertainties in morphological classification should be taken into account when making any firm statement. Galaxies in dense environments can have their morphology distorted by tidal effects which makes them difficult to classify. One should refer to Appendix A (only available in electronic form) in order to visually check the morphology of each individual galaxy in more detail. We also refer to the detailed morphological classification taken from RC3 presented in Table 1 which gives a general idea on the complexity of the morphologies.

In Table 3 we give both the $\mathrm{CO}(1-0)$ and $\mathrm{CO}(2-$ 1) integrated line intensitites. In order to estimate the $\mathrm{CO}(2-1) / \mathrm{CO}(1-0)$ intensity ratios we need to convolve the $\mathrm{CO}(2-1)$ data to the same angular resolution as the $\mathrm{CO}(1-0)$ data. Since we observed only a single position for most galaxies, we can not do this. However, taking the values in Table 3 at face value, the average $\mathrm{CO}(1-0)$ to $\mathrm{CO}(2-1)$ line intensity ratio is $0.93 \pm 0.47$. This is an upper limit to the line ratio. In the case of a molecular gas distribution more extended than both the $\mathrm{CO}(1-0)$ and $\mathrm{CO}(2-1)$ telescope beams ( $45^{\prime \prime}$ and $23^{\prime \prime}$, respectively), the correction for different angular resolutions would be 1.0.
In the other extreme, with the $\mathrm{CO}$ emission originating in a point source, the correction for different angular resolutions would be 0.25 . Since our telescope beam in almost all cases is large with respect to the optical extent of the galaxies, and since the molecular gas is likely to be centrally concentrated, the correction for different angular resolutions should be $\sim 0.5$. Our average line ratio is thus $\sim 0.5 \pm 0.4$. This value is lower than that found by Braine et al. (1993) of $0.89 \pm 0.34$ for normal spiral galaxies. The lower value is characteristic of optically thick and subthermally excited molecular gas and most likely reflects the lower star formation activity in our environmentally selected sample as opposed to far infrared bright selected samples.

In Fig. 4 we verify that the HDS and CS are also very similar to the galaxies in other samples in terms of SFE. We conclude that the intermediate type spirals in the HDS and CS do not belong to a separate class of objects but contain objects with properties similar to galaxies in clusters, nearby spiral galaxies and infrared luminous galaxies.

\section{Summary}

In this paper we present millimetric and optical data obtained in order to study environmental effects in galaxies. Our sample has 47 intermediate Hubble type spirals in either dense environments or in the field. We compared general properties, such as far-infrared luminosity, blue luminosity, and total molecular gas content, to other samples of galaxies, such as ultraluminous infrared galaxies, clusters of galaxies and spiral galaxies. We find that overall our sample has general properties very similar to these other galaxies; i.e. they are not a separate class of objects. 
Acknowledgements. We thank the ON team of observers at the ESO1.52 m, in particular Christopher Willmer for helping with the data reduction and Henrique Schmitt for valuable suggestions regarding the stellar contamination. This research has made use of the NASA/IPAC Extragalactic Database (NED) which is operated by the Jet Propulsion Laboratory, California Institute of Technology, under contract with the National Aeronautics and Space Administration. The Digitized Sky Surveys were produced at the Space Telescope Science Institute under US Government grant NAG W-2166. The images of these surveys are based on photographic data obtained using the Oschin Schmidt Telescope on Palomar Mountain and the UK Schmidt Telescope. D.F.M. was partially supported by CNPq Fellowship 301456/95-0, and the Swedish Vetenskapsrådet project number F620-489/2000. M.A.G.M. was supported by CNPq grant 301366/86-1. T.W. was supported by Vetenskapsrådet project number F1299/1999.

\section{References}

Aaronson, M., Huchra, J., Mould, J., Schechter, P. L., \& Tully, R. B. 1982, ApJ, 258, 64

Andreani, P., Casoli, F., \& Gerin, M. 1995, A\&A, 300, 43

Baldwin, J. A., Phillips, M. M., \& Terlevich, R. 1981, PASP, 93,5

Bica, E. 1988, A\&A, 195, 76

Braine, J., Combes, F., Casoli, F., et al. 1993, A\&AS, 97, 88

Cardelli, J. A., Clayton, G. C., \& Mathis, J. S. 1989, ApJ, 345, 245

Casoli, F., Boisse, P., Combes, F., \& Dupraz, C. 1991, A\&A, 249,359

Casoli, F., Sauty, S., Gerin, M., et al. 1998, A\&A, 331, 451

Cid Fernandes, R. Jr., Storchi-Bergmann, T., \& Schmitt, H. R. 1998, MNRAS, 297, 579

Combes, F., Prugniel, P., Rampazzo, R., \& Sulentic, J. W. 1994, A\&A, 281, 725

Coziol, R., Iovino, A., \& de Carvalho, R. R. 2000, AJ, 120, 47

da Costa, L. N., Pellegrini, P. S., Willmer, C., et al. 1989, AJ, 97,315

de Mello, D. F., Wiklind, T., \& Maia, M. A. G. 2002, A\&A, 381, 771 (Paper II)

de Vaucouleurs, G., de Vaucouleurs, A., Corwin, H. G., Jr., et al. 1991, Third Reference Catalogue of Bright Galaxies (RC3) (New York: Springer)
Dressler, A. 1980, ApJ, 236, 351

Elfhag, T., Booth, R. S., Hoeglund, B., Johansson, L. E. B., \& Sandqvist, A. 1996, A\&AS, 115, 439

Forveille, T., Guilloteau, S., \& Lucas, R. 1990, IRAM internal report

Hickson, P. 1982, ApJ, 255, 382

Ho, L. C., Filippenko, A. V., \& Sargent, W. L. W. 1997, ApJ, 112,315

Horellou, C., Casoli, F., Combes, F., \& Dupraz, C. 1995, A\&A, 298,743

Horellou, C., \& Booth, R. 1997, A\&AS, 126, 3

Huchra, J. P., \& Geller, M. J. 1982, ApJ, 257, 423

Lauberts, A. 1982, The ESO/Uppsala Survey of the ESO(B) Atlas (Munich: European Southern Observatory)

Lauberts, A., \& Valentijn, E. A. 1989, The Surphace Photometry Catalogue of the ESO-Uppsala Galaxies (Garching bei München: ESO)

Leon, S., Combes, F., \& Menon, T. K. 1998, A\&A, 330, 37

Lonsdale, C., \& Helou, G. 1985, Cataloged galaxies and quasars observed in the IRAS survey, Pasadena: Jet Propulsion Laboratory (JPL)

Maia, M. A. G., da Costa, L. N., \& Latham, D. W. 1989, ApJS, 69,809

Maia, M. A. G., Pastoriza, M. G., Bica, E., \& Dottori, H. 1994, ApJSS, 93, 425

McCall, M. L., Rybski, P. M., \& Shields, G. A. 1985, ApJS, 57,1

Moshir, M., Kopar, G., Conrow, T., et al. 1990, IRAS Faint Source Catalogue, version 2.0.

Roberts, M. S., \& Haynes, M. P. 1994, ARA\&A, 32, 115

Rubio, M., Garay, G., Montani, J., \& Thaddeus, P. 1991, ApJ, 368,173

Sage, L. J. 1993, A\&AS, 100, 537

Sanders, D. B., Scoville, N. Z., \& Soifer, B. T. 1991, ApJ, 370, 158

Sanders, D. B., \& Mirabel, I. F. 1996, ARA\&A, 34, 749

Sanroma, M., \& Salvador-Solé, E. 1990, ApJ, 360, 16

Storchi-Bergmann, T., Calzetti, D., \& Kinney, A. L. 1994, ApJ, 429, 572

Young, J. S., Shuding, X., Kenney, J. D. P., et al. 1989, ApJS, 70,699

Veilleux, S., \& Osterbrock, D. E. 1987, ApJS, 63, 295

Whitmore, B. C., \& Gilmore, D. M. 1991, ApJ, 367, 64

Willmer, C. N. A., Maia, M. A. G., Mendes, S. O., et al. 1999, AJ, 118, 1131

Zabludoff, A. I., \& Mulchaey, J. S. 1998, ApJ, 496, 39 